\title{
4. Inhibitory Effects of Caffeine on Contraction of Depolarized Smooth Muscle
}

\author{
By Shigeru YamashiTA \\ Department of Pharmacology, Faculty of Medicine, University of Tokyo, \\ Bunkyo-ku, Tokyo 113 \\ (Communicated by Setsuro EBASHI, M. J. A., Feb. 12, 1992)
}

\begin{abstract}
By measuring the isometric tension produced by thin strips of taenia coli smooth muscle, inhibitory effects of caffeine on the acetylcholine (ACh)-induced phasic contraction were examined under the high-K depolarized state. Caffeine $(2 \mathrm{mM})$ inhibited ACh-contraction by three different mechanisms: 1) by decreasing the efficiency of Ca-release from Ca-store, 2) by inhibiting the loading of Ca-store with $\mathrm{Ca}^{2+}$ and 3) by accelerating the depletion of Ca-store. In the presence of physiological concentration of $\mathrm{Ca}^{2+}$ the first mechanism was found to be dominant with a slight contribution of the second. Under the nominally Ca-free conditions the contribution of the third was also important.
\end{abstract}

Key words: Smooth muscle; caffeine; pharmacomechanical coupling; guinea pig; taenia coli.

Introduction. Caffeine is known to have dual effects on the contraction of the smooth muscle: induction of contraction by potentiation of Ca-induced Ca-release (CICR) in vascular and visceral smooth muscles as in skeletal and cardiac muscles, ${ }^{1), 2}$ and inhibition of high-K, drug-induced and spontaneous contractions in the smooth muscle. ${ }^{3), 4)}$ The latter mechanism is much less understood than the former.

Recent studies on the inhibitory action of caffeine showed that caffeine inhibits high-K and agonist-induced tonic contractions in taenia coli and aorta by decreasing the entry of $\mathrm{Ca}^{2+}$ into the cell and that the drug inhibits the phasic phase of noradrenaline-induced contraction by inhibition of Ca-release from the Ca-store. ${ }^{5), 6)}$ It is also shown that caffeine directly inhibits the contractile protein system in uterine, aortic and gizzard smooth muscles. $^{7)-9)}$

In the present study the inhibitory effect of low concentration of caffeine on the phasic phase of acetylcholine ( $\mathrm{ACh}$ )-induced contraction in high- $\mathrm{K}$ depolarized muscle was examined by the use of thin strips of guinea-pig taenia coli.

In the following description "ACh-contraction" always indicates "the phasic phase of ACh-induced contraction in the high-K depolarized state".

Methods. Thin strips of smooth muscle, $150-250 \mu \mathrm{m}$ in diameter and 1-2 mm in length, were prepared from taenia coli of male guinea pigs and isometric tension was recorded as described previously. ${ }^{10)}$ Locke's solution of the following composition was used as the standard solution; $154 \mathrm{mM} \mathrm{NaCl}, 5.4 \mathrm{mM} \mathrm{KCl}, 1.8 \mathrm{mM} \mathrm{NaHCO}_{3}, 1.8 \mathrm{mM} \mathrm{CaCl}_{2}$, $1.8 \mathrm{mM} \mathrm{MgCl} \mathrm{Mg}_{2}$ and $5.6 \mathrm{mM} \mathrm{D}$-glucose. ACh $\left(10^{-5} \mathrm{~g} / \mathrm{ml}\right)$ was applied in the isotonic K-Locke's solution in which the whole $\mathrm{Na}^{+}$in Locke's solution was replaced by equimolar $\mathrm{K}^{+}$or in Ca-free K-Locke's solution which had the same composition as K-Locke's solution except that $\mathrm{CaCl}_{2}$ was omitted and washed after 2 min. Solutions were saturated with air and maintained at $25^{\circ} \mathrm{C}$.

Results. In all the experiments thin strips of taenia coli with the diameter ranging $150-250 \mu \mathrm{m}$ were used to minimize the difrusion time. In the thin strips, the ratio of the 


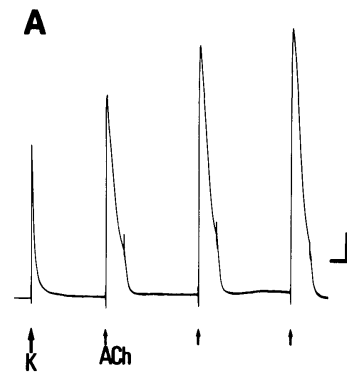

B

Fig. 1. Inhibitory effects of caffeine on ACh-contraction. Traces in each figure (Figs. 1-3) were recorded with the same muscle strip. Horizontal and vertical calibration bars denote $2 \mathrm{~min}$ and $10 \mathrm{mg}$, respectively, as in all the other figures. After Locke's solution was replaced with K-Locke's solution (at " $\mathrm{K}$ " in A), ACh $\left(10^{-5} \mathrm{~g} / \mathrm{ml}\right)$ was repeatedly applied for 2 min beginning at each thin arrow (A, B). When the amplitude of ACh-contraction became constant, $2 \mathrm{mM}$ caffeine was applied during the period indicated with a bar (B). Contraction at the beginning of the bar in B indicates caffeine contraction.

sustained tonic tension to the maximal phasic tension was below $10 \%$, smaller than that in the conventional thick preparation with the diameter ranging $1-2 \mathrm{~mm}$. In the latter preparation the ratio was $25-50 \%$.

When the ACh-contraction was repeated in high- $\mathrm{K}$ solution, the amplitude of the contraction increased gradually (Fig. 1A) until it attained to a constant level. As shown in Fig. 1B, ACh-contraction was strongly inhibited by the addition of a relatively low concentration of caffeine $(2 \mathrm{mM})$ and the amplitude was reduced to about $40 \%$. The inhibitory effect of caffeine became greater at higher concentrations, and $10 \mathrm{mM}$ caffeine completely abolished the ACh-contraction (data not shown). In most cases, the lowest concentration of caffeine which clearly showed inhibition was between 1 and $2 \mathrm{mM}$.

The inhibitory effects of caffeine were further examined by "loading" experiments. The ACh-contraction was repeated in Ca-free solution before each part of the experiments (A-F in Fig. 2 and A-E in Fig. 3) until the contraction was virtually abolished (first arrow in each figure). Then $\mathrm{Ca}^{2+}$ was applied again to the bathing solution for $6 \mathrm{~min}$. This procedure will be called "loading" because the Ca-store is considered to be loaded with $\mathrm{Ca}^{2+}$ during this procedure. After the loading, the muscle strip was incubated again in Ca-free solution for various periods of time, and then the ACh-contraction was induced under Ca-free solution (second arrow).

As shown in Fig. 2, the presence of $2 \mathrm{mM}$ caffeine during the loading slightly reduced the ACh-contraction induced after removal of $\mathrm{CaCl}_{2}$ (Fig. 2B). The extent of reduction was about $10 \%$, which is far less than the inhibition under the presence of caffeine observed in the experiment in Fig. 1. When $2 \mathrm{mM}$ caffeine was continuously present during the loading and the ACh-contraction (Fig. 2C), the amplitude of the contraction was reduced by $65 \%$ (Fig. 2C), which was comparable with the extent of inhibition shown in Fig. 1. Since the loading process was the same in both cases (Figs. $2 \mathrm{~B}$ and $2 \mathrm{C}$ ), the difference suggests that caffeine greatly decreased the efficiency of Ca-release induced by ACh. The possibility that caffeine depleted the Ca-store during 2 min prior to the application of ACh is excluded by the result in Fig. 2D, because even after 2 min incubation in Ca-free solution with caffeine, the ACh-contraction with about $80 \%$ of the amplitude in the control was induced (Fig. 2D), which means that enough amount of $\mathrm{Ca}^{2+}$ was still present in the store. Therefore, the presence of caffeine greatly reduced the contraction in spite of enough amount of $\mathrm{Ca}^{2+}$ in 

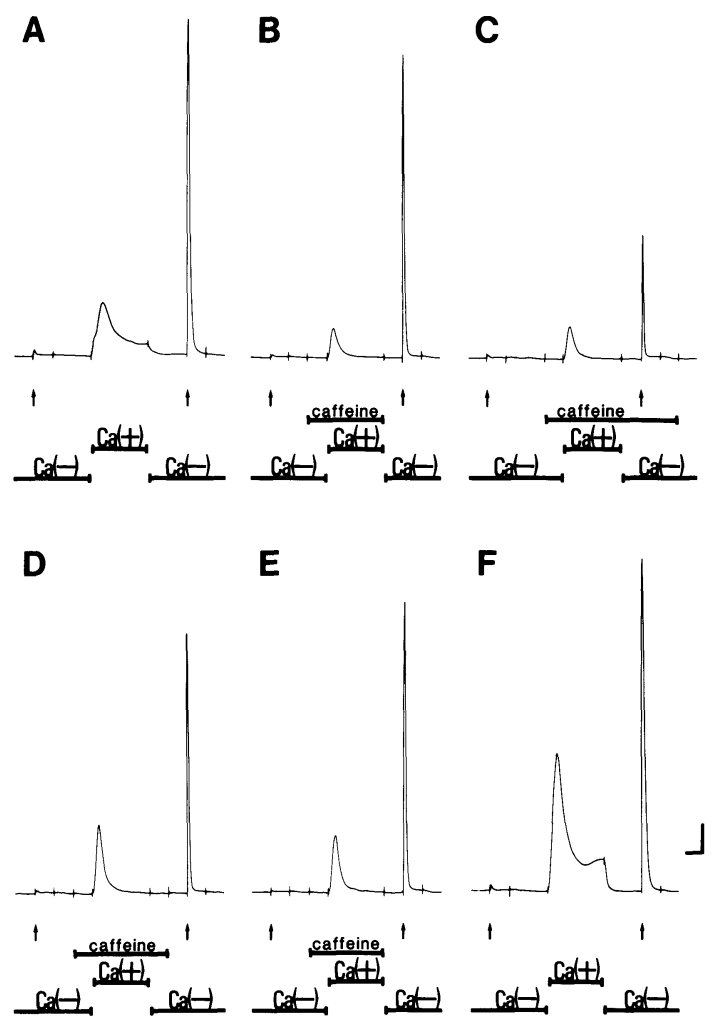

Fig. 2. Inhibitory effects of caffeine on ACh-induced Ca-release and on loading of Ca-store. ACh $\left(10^{-5} \mathrm{~g} / \mathrm{ml}\right)$ was applied for $2 \mathrm{~min}$ in Ca-free K-Locke's solution at each arrow. Bars denoted as " $\mathrm{Ca}(-)$ " or " $\mathrm{Ca}(+)$ " indicate the periods during which $\mathrm{Ca}^{2+}$ was nominally free or $1.8 \mathrm{mM}$ $\mathrm{CaCl}_{2}$ was present in the external solution. During tonic phase of $\mathrm{K}$-contracture, ACh-contraction was repeatedly induced in K-Locke's solution and $\mathrm{CaCl}_{2}$ was then removed (not shown). Before the period shown in each figure, A-F, ACh-contraction was repeated in Ca-free K-Locke's solution until the contraction was virtually abolished (first arrow in A-F). The bars with the notation "caffeine" indicate the periods during which $2 \mathrm{mM}$ caffeine was applied. A, F: control. B, E: Loading was performed in the presence of $2 \mathrm{mM}$ caffeine. See also text.

the store. In Fig. 2 the same experimental procedure was repeated twice (B and E, A and F) for examining the possible change in the loading during the experiment. By comparison of Fig. $2 \mathrm{~B}$ with $2 \mathrm{E}$ and of Fig. $2 \mathrm{~A}$ with $2 \mathrm{~F}$, the efficiency of the loading in the presence or absence of caffeine was observed to remain unchanged throughout the experiment.

The loading experiments also revealed that caffeine has depleting effect on the Ca-store. In Fig. 3, the muscle strip was incubated for $10 \mathrm{~min}$ in Ca-free solution after the loading for $6 \mathrm{~min}$ and then, the ACh-contraction was induced. The presence of $2 \mathrm{mM}$ caffeine during the loading and additional 6 min reduced the contraction by more than $75 \%$ (Fig. 3C), whereas the presence of caffeine only during the loading diminished the contraction only by $15 \%$ (Fig. 3B). Since the loading process was the same, the rapid depletion must have taken place in the former case during the 6 min after the loading. Thus, $2 \mathrm{mM}$ caffeine was also shown to accelerate the depletion of the Ca-store. 

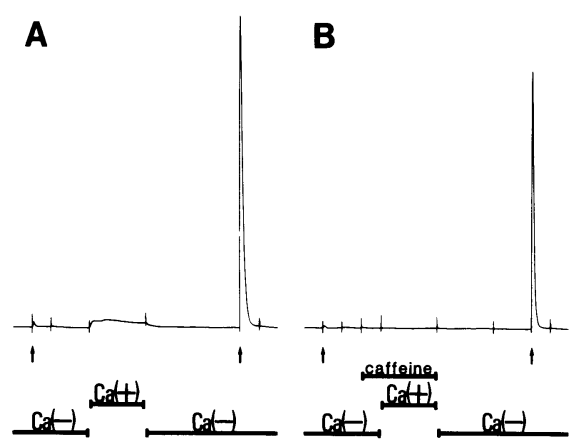

C

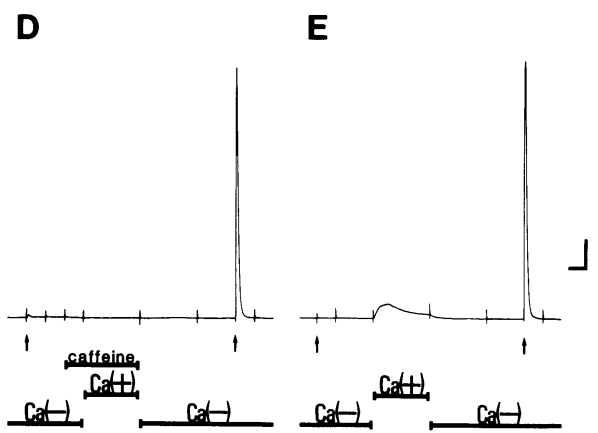

Fig. 3. Accelerated depletion of Ca-store by caffeine. Notations and basic experimental procedures are the same as in Fig. 2. ACh-contraction was induced 10 min after loading (second arrow in A-E). A, E: control. B, D: Loading was performed in the presence of $2 \mathrm{mM}$ caffeine. See also text.

Discussion. The phasic phase of ACh-contraction in various types of the smooth muscle is generally considered to be induced by Ca-release from the intracellular Ca-store ${ }^{11), 12)}$ presumably sarcoplasmic reticulum (SR). Since the amount of released $\mathrm{Ca}^{2+}$ is determined not only by the amount of stored $\mathrm{Ca}^{2+}$ but also by the efficiency of Ca-release, inhibitory effects of the drugs on ACh-contraction can be exerted by either of the three mechanisms: (i) by decreasing the efficiency of Ca-release, (ii) by decreasing the amount of $\mathrm{Ca}^{2+}$ in the Ca-store either by inhibition of loading or by acceleration of depletion or (iii) by decreasing the Ca-sensitivity of the contractile protein system.

The contribution of the last mechanism to the inhibitory action of caffeine shown in this report is expected to be very slight for two reasons. First, according to Kyozuka ${ }^{7)}$ and Ozaki et al. ${ }^{8)}$ showing that caffeine dose-dependently inhibited the contractile protein system in the chemically skinned fiber of uterine and gizzard smooth muscles, $3 \mathrm{mM}$ caffeine reduced the tension by only $10 \%$, although higher concentrations of caffeine $(>10 \mathrm{mM})$ showed stronger inhibition. If the dose-dependence of the inhibitory action of caffeine on the contractile protein system in taenia coli is much the same as in uterine or gizzard smooth muscle, caffeine is expected to have only slight inhibitory effect on the contractile protein system at least at the low concentration used in this report. Second, if caffeine inhibits ACh-contraction mainly by the decrease of the sensitivity of the contractile protein system, contraction induced in various ways must be inhibited to the same extent, irrespective of the mode of induction. However, electrically induced contraction in depolarized state (EICD) was hardly inhibited by low concentration of caffeine, whereas ACh-contraction was strongly inhibited by the same concentration of the 
drug. ${ }^{10)}$

The present work showed that caffeine, at relatively low concentration $(2 \mathrm{mM})$, inhibited ACh-contraction of taenia coli and that the inhibitory action of the drug could be caused by three different mechanisms at least: 1) by decreasing the efficiency of Ca-release, 2) by the inhibition of loading of Ca-store and 3) by accelerating the depletion of Ca-store.

The first mechanism was demonstrated by the loading experiments showing that ACh-contraction elicited in the presence of caffeine was much weaker than that elicited in the absence of the drug despite nearly equal amount of stored $\mathrm{Ca}^{2+}$ in both cases (Fig. 2). An alternative interpretation may be that caffeine reduced the sensitivity of the contractile protein system to $\mathrm{Ca}^{2+}$. However, significant contribution of this mechanism is unlikely as discussed above. The inhibition of loading of the Ca-store by $2 \mathrm{mM}$ caffeine was slight, with the reduction of the amplitude of ACh-contraction only by about $10 \%$ (Fig. 2, B and E). It is too slight to explain the strong inhibition of ACh-contraction by caffeine shown in Fig. 1. Acceleration of depletion of the Ca-store was demonstrated by the rapid decrease in the amount of stored $\mathrm{Ca}^{2+}$ when $\mathrm{CaCl}_{2}$ was removed in the presence of caffeine (Fig. 3). The depleting effect of caffeine was not observed in the presence of $1.8 \mathrm{mM} \mathrm{CaCl}_{2}$ since loading of the Ca-store occurred readily in this condition. Therefore, in the presence of the standard concentration of $\mathrm{Ca}^{2+}(1.8 \mathrm{mM})$ the inhibition of ACh-contraction by $2 \mathrm{mM}$ caffeine is mainly due to the decrease in the efficiency of Ca-release, though inhibition of loading of Ca-store by caffeine may slightly contribute. In Ca-free solution the depletion of the Ca-store can be important as a main mechanism of inhibitory action of caffeine.

The most important conclusion of the present study is that caffeine inhibits ACh-contraction by decreasing the efficiency of Ca-release and that this mechanism can be dominant over the inhibitory mechanisms decreasing the amount of $\mathrm{Ca}$ in Ca-store. To demonstrate decrease in the efficiency of Ca-release by caffeine it is important to separate this effect from the effects on the amount of stored $\mathrm{Ca}^{2+}$. In loading experiments the drugs are usually applied after loading, but this procedure was inappropriate for caffeine because Ca-release by caffeine would decrease the amount of stored $\mathrm{Ca}^{2+}$. This difficulty was avoided by loading in the presence of caffeine. To minimize the inhibition of loading and depletion of Ca-store by caffeine a low concentration of the drug was used.

Since the ACh-contraction was induced in fully depolarized muscle in the present experiments, ${ }^{12)}$ the ACh-induced Ca-release is not mediated by membrane potential change, but seems to occur through the pathway of inositol 1,4,5-trisphosphate $\left(\mathrm{IP}_{3}\right)$ as the second messenger. ${ }^{13), 14)} \mathrm{IP}_{3}$ is known to induce Ca-release from SR by opening the Ca-channels on the SR membrane in smooth muscles including taenia coli. ${ }^{15)-18)}$ Although caffeine is known to open the Ca-channel used for CICR, ${ }^{19), 20)}$ this mechanism does not explain the decrease in the efficiency of ACh-induced Ca-release by caffeine because the caffeine-sensitive Ca-channel is a different type of the Ca-channel from that opened by $\mathrm{IP}_{3} \cdot{ }^{16), 21)}$ The decrease in the efficiency of Ca-release by caffeine may be due to the inhibition of either $\mathrm{IP}_{3}$-formation or $\mathrm{IP}_{3}$-induced Ca-release.

Acknowledgements. I sincerely thank Profs. S. Ebashi, M. J. A., and Y. Nonomura for their valuable discussion and encouragement. This work was supported by grants from the Ministry of Education, Science and Culture, Japan, from the Hoansha Foundation, from the Fugaku Trust for Medicinal Research and from the Iatrochemical Foundation.

\section{References}

1) Endo, M.: Proc. Japan Acad., 51, 479-484 (1975).

2) Iino, M.: J. Gen. Physiol, 94, 363-383 (1989). 
3) Ito, Y., and Kuriyama, H.: J. Gen. Physiol., 57, 448-463 (1971).

4) Somlyo, A. V., and Somlyo, A. P.: J. Pharmacol. Exp. Ther., 159, 129-145 (1968).

5) Ahn, H. Y. et al.: Br. J. Pharmacol., 93, 267-274 (1988).

6) Leijten, P. A. A., and van Breemen, C.: J. Physiol., Lond., 357, 327-339 (1984).

7) Kyozuka, M.: Biomedical Res., 4, 523-532 (1983).

8) Ozaki, H. et al.: Naunyn-Schmiedeberg's Arch. Pharmacol., 341, 262-267 (1990).

9) Sato, K. et al.: ibid., 338, 443-448 (1988).

10) Yamashita, S., and Nonomura, Y.: Biomedical Res., 2, 69-72 (1981).

11) Ohashi, H. et al.: Japan. J. Pharmacol., 24, 601-611 (1974).

12) Schild, H. O.: Proc. 2nd. Intern. Pharmacol. Meeting (ed. E. Bulbring). vol. 6, Pergamon Press, Oxford, pp. 95-104 (1964).

13) Berridge, M. J.: Ann. Rev. Biochem., 56, 159-193 (1987).

14) Berridge, M. J., and Irvine, R. F.: Nature, 312, 315-321 (1984).

15) Hashimoto, H., et al.: J. Physiol., Lond., 370, 605-618 (1986).

16) Iino, M.: Biochem. Biophys. Res. Commun., 142, 47-52 (1987).

17) Somlyo, A. V. et al.: Proc. Natl. Acad. Sci. U.S.A., 82, 5231-5235 (1985).

18) Suematsu, E. et al.: Biochem. Biophys. Res. Commun., 120, 481-485 (1984).

19) Endo, M.: Curr. Top. Membr. Transp., 25, 181-230 (1985).

20) Smith, J. S. et al.: Nature, 316, 446-449 (1985).

21) Van Breemen, C., and Saida, K.: Ann. Rev. Physiol., 51, 315-329 (1989). 